\title{
Microwave Produced Tannin-furanic Foams
}

\author{
Christian Kolbitsch ${ }^{1}$, Martin Link ${ }^{1}$, Alexander Petutschnigg ${ }^{1}$, Stefanie Wieland ${ }^{1} \&$ Gianluca Tondi $^{1}$ \\ ${ }^{1}$ Wood Technology Department, Salzburg University of Applied Sciences 5431, Kuchl, Austria \\ Correspondence: Gianluca Tondi, Wood Technology Department, Salzburg University of Applied Sciences 5431, \\ Kuchl, Austria. Tel: 43-50-2211-2250. E-mail: gianluca.tondi@fh-salzburg.ac.at
}

Received: March 30, 2012 Accepted: April 22, 2012 Online Published: June 11, 2012

doi:10.5539/jmsr.v1n3p84 URL: http://dx.doi.org/10.5539/jmsr.v1n3p84

\begin{abstract}
A new method to produce formaldehyde-free tannin-based furanic foams has been developed. The use of microwaves as energy source allows homogeneous tannin foams to be produced in a very short time. In this paper the main characteristics of these foams are investigated and compared to the state of the art. It has been seen that the microwave production allows a high degree of repeatability in terms of bulk density. The microwave-produced foams have shown similar features to the previous tannin-furanic foams produced in the hot-press or in the oven. The high water affinity of these materials has allowed the study of partial recovering of the catalystwhile the increased fire resistance of the tannin-furanic foams leads to the assumption that there is an important effect onthe degree of polymerization in the fire resistance of these innovative, natural materials.
\end{abstract}

Keywords: tannin, furanic, natural polymer, foam, microwave

\section{Introduction}

In the last few years, the tannin-based rigid foams were obtained from $95 \%$ natural substrates. These foams were already known for their application as floral foams (Merkleham \& Pizzi, 1994) but more recent studies have shown that they can also be used in metal ion adsorption (Tondi et al., 2009) and as internal insulating materials for interior and exterior wooden doors (Tondi et al., 2008).

Unfortunately these foams were not completely natural because the contribution of formaldehyde (around 5\%) was necessary to set up a stable polymer.

The completely natural version of these foams has been recently developed in the laboratories of the Salzburg University of Applied Sciences (Link et al., 2011).

The skeleton of these advanced foams is $100 \%$ natural because it is made by the condensation between $60-65 \%$ of tannin extract and $35-40 \%$ of furfuryl alcohol. These two components are both natural: Furfuryl alcohol is obtained throughout catalytic reduction of furfural (Nagaraja et al., 2003), which is obtained by hydrolysis of the hemicelluloses from several agricultural crops (Aguilar et al., 2002); while flavonoid tannins are vegetal products obtained by water extraction of bark or wood chips (Sealy-Fisher \& Pizzi, 1992).

From the viewing angle of mechanical properties the production method has a significant influence.

The formaldehyde-free advanced foams, indeed, were developed carrying external energy through the hot plates of a press while the formaldehyde-enriched formulations were self-blowing at room temperature. These different processing methods are affecting the physical properties of the foam and the comparison could be inappropriate. Anyhow, the compressive strength of foams with similar densities showed that the formaldehyde reinforced foams are generally more resistant.

The foaming process to obtain tannin-based rigid foams consists of three phases: mixing, expansion and curing. The components of the blend (tannin extract, furfuryl alcohol, blowing agents, and additives) are mechanically stirred until the system is homogeneous.

The expansion phase starts as soon as a catalyst (sulfuric acid) is added to the mix and some external energy is applied to the system. In this phase the polycondensation of the resin and the evaporation of the blowing agent take place simultaneously. This causes the whole mix to blow and cure at the same time. The last phase is taken up with the hardening of the tannin-furanic network which stabilizes the foam in a few minutes. 
The use of microwaves as energy carrier systemin wet chemical synthesis allows to improve significantly the curing time and the yield of the reaction (Shi \& Hwang, 2003). This is due to a more efficient heat-transfer that allows the reaction to achieve the kinetic activation energyin a very limited time. The idea to use microwaves for the production of tannin-furanic foams offer an alternative method to produce natural foams that could be more suitable for industrial production plants.

In this paper, an overview of the main physical and mechanical characteristicsof microwave-produced formaldehyde-free tannin-furanic foams is presented. These properties are compared with the previously studied tannin-furanic foams (Tondi \& Pizzi, 2009; Tondi et al., 2009; Link et al., 2011). Mechanical-, moisture-,water and fire behaviour are investigated.

\section{Experimental}

\subsection{Materials}

Mimosa (Acacia mearnsii, formerly mollissima de Wildt) tannin extract was provided by Silva Chimica (Italy). Furfuryl alcohol was kindly supplied by Transfurans chemicals (Belgium). The other chemicals such as: methanol (99\%), diethylether (98\%) and sulfuric acid (32\%) were supplied by Carl Roth and Merck.

\subsection{Foam Preparation}

\subsubsection{Formulation and Procedure}

Furfuryl alcohol, water, methanol and diethylether were manually mixed to a homogeneous liquid in 500ml glass beakers. The tannin powder was added and mixed until a homogeneous, viscous blend was achieved. Finally the catalyst was poured and the blend was stirred again. The homogeneous suspension was then transferred into a box made of HDF (High density fibreboard) glued with PVAc (Polyvinylacetate) with the dimensions $10 \times 10 \times 2.5 \mathrm{~cm}$. The box was covered with another layer of HDF and a load was placed on top to prevent the foam from uncovering the reaction box. The blend was blown in a Whirlpool M515 microwave oven at the frequency of $2450 \mathrm{MHz}$ with a power of $600 \mathrm{~W}$ and a processing time of 2.0 minutes. The detailed quantities for the production of the three foams are reported in Table 1.

Table 1. Formulations of the microwave-produced tannin-furanic foams

\begin{tabular}{llllllll}
\hline Sample & $\begin{array}{l}\text { Tannin } \\
(\%)\end{array}$ & $\begin{array}{l}\text { Furfuryl } \\
\text { alcohol } \\
(\%)\end{array}$ & $\begin{array}{l}\text { Water } \\
(\%)\end{array}$ & $\begin{array}{l}\text { Methanol } \\
(\%)\end{array}$ & $\begin{array}{l}\text { Diethylether } \\
(\%)\end{array}$ & $\begin{array}{l}\mathrm{H} 2 \mathrm{SO} 4 \\
(\%)\end{array}$ & $\begin{array}{l}\text { Density } \\
{\left[\mathrm{Kg} / \mathrm{m}^{3}\right]}\end{array}$ \\
\hline A & 42 & 26 & 8 & & 6 & 18 & 110 \\
B & 52 & 18 & & 7 & & 23 & 150 \\
C & 42 & 23 & 17 & & & 18 & 224 \\
\hline
\end{tabular}

\subsubsection{Seasoning and Cutting}

After blowing the foam, the boxes were cut into samples of $50 \times 50 \mathrm{~mm}$ each. The sandwich panels with the dark sponge-like core were left for 2 to 3 days at $20^{\circ} \mathrm{C}$ and $65 \% \mathrm{RH}$ to allow the evaporation of the residual blowing agent trapped in the foams.

\subsection{Testing Method}

\subsubsection{Bulk Density}

The density $(\rho)$ is the ratio between mass $(m)$ and volume $\left(l^{*} w^{*} h\right)$ of the whole sample after removal of the top panels.

$$
\rho=\frac{m}{(l * w * h)}
$$

The density was measured for a minimum of 12 samples for each formulation.

\subsubsection{Density Profile}

The density profiles of $15 \mathrm{~mm}$ foam slices were measured after cutting out the external HDF layers to evaluate the homogeneity of the core. The density profile measurements were performed using an EWS Dense-lab X. The step rate between 0.1 and $0.2 \mathrm{~mm}$ was selected according to the resolution of the instrument. 


\subsubsection{Compression Resistance}

These tests were performed using pieces cut into 50x50x20mm and proceeded according to the DIN 52185 (1976). The tests were carried out in a Zwick-Roell Z250. 12 samples of each formulation were tested along the direction of growth of the foam applying a compression rate of $2 \mathrm{~mm} / \mathrm{min}$. The test ended when the sample reached $75 \%$ of their original height.

The maximum compressive strength (or fracture stress) was evaluated with Formula 2 according to the standard evaluated at the moment in which the elastic behaviour of the foam reached its maximum (collapse of the first layers of cells).

$$
\sigma_{c p}=\frac{F_{\max }}{A}=\left[\frac{N}{m m^{2}}\right]
$$

\subsubsection{Behaviour with Moisture}

The microwave-produced tannin-furanic foams were cut in 50x50x20mm samples and were subjected to different moisture conditions keeping the fixed temperature of $20^{\circ} \mathrm{C}$. The previously dried samples were kept at $40,65,80$ and $95 \%$ relative humidity for at least 72 hours for each humidity state. The weight of the sample was monitored to control the absorption of water.

\subsubsection{Forced and Natural Water Absorption}

Two dipping methods were applied to understandthe absorption process of the tannin foams: The forced absorption was carried out according to the EN 317 (1993). In contrast, thenatural absorption was performed laying the samples on the water surface. The tests were always done with 5 samples $(50 \times 50 \times 20 \mathrm{~mm})$ of each formulation. The weight of the samples was monitored after 2, 8, 24 and 48 hours and the water content was calculated with Formula 3.

$$
G_{t}=\frac{m_{2}-m_{1}}{m_{1}} * 100
$$

\subsubsection{Catalyst Recovery}

The 50x50x20mm samples were dipped in a beaker containing $200 \mathrm{ml}$ of water. The samples were forcedly kept under water with a load. A vacuum of $50 \mathrm{mbar}$ for 6.5 minutes was applied to shorten the penetration of water in the foam cells. Afterwards, the samples were removed and the $\mathrm{pH}$-value of the water solution was measured with a WTW inolab $\mathrm{pH}$-meter. This leaching process was repeated four times. The amount of sulfuric acid was indirectly calculated considering the complete dissociation of the acid.

\subsubsection{Fire Properties}

The center of the 50x50mm face of the sample without HDF layer was laid on a metal grid $5 \mathrm{~cm}$ above a $100 \%$ conical flame of a Bunsen burner (max. $\mathrm{O}_{2}$ consumption). The flame height without sample was approximate $12 \mathrm{~cm}$. The samples were exposed to the flame for $60 \mathrm{sec}$ and allowed to self-extinguish afterwards. The self-extinguish time is the time required for the sample to completely stop the glowing. 5 samples per foam were tested in order to assess self-extinguish time and weight loss.

\section{Results and Discussion}

Formaldehyde-free tannin-furanic foams were prepared in a microwave oven. The highly efficient heat transfer of the microwaves allows the hardening of the polymer and the blowing of the solvent in a shorter time than the hot-press procedure. The microwave irradiation, indeed, produces high energy in the wet blend and induces a much faster polymerization of the resin. The time and the temperature locally reached play an important role in the condensation of tannin with furfuryl alcohol. In facts, the polymer cured with the energy of microwaves requires lower time for completing the polymerization and the network of the foam appears to bestiffer.

The use of microwaves allows also faster water evaporation. For this reason lower amount of organic blowing agentsare required and consequent less or no recovery of these solvents has to be planned in the up-scaling of the production to industrial plants.

The microwaves-produced foam samples were subjected to density studies, tests relating to mechanical properties, water affinity and behaviour towards fire, all of which are described in the following chapters. Additionally, a further interesting study on the possibility of catalystrecovery was performed. 


\subsection{Density and Homogeneity}

The equilibrium between the polymerization reaction and the evaporation of the solvents is responsible for the final density of the foam (Tondi \& Pizzi, 2009).

The properties of the foams are highly dependent on the formulation and on the conditions applied during the blowing process. The lab-scale production of self-blowing foams is always subject to many parameters that can affect the blowing and the derived product of two identical formulations can carry to slightly different foams. Hence, the use of microwavesas energy carriersystem will also significantly influence the properties of the foam.

In Figure 1, the core images of the microwave-produced tannin-furanic foams are reported. The pictures show that the lab-made foams produced with the microwave system are homogeneous: only some cells have a diameter larger than $1 \mathrm{~mm}$ and the frame appearance was also encouraging. For this reason, density profile measurements were carried out (Figure 2).
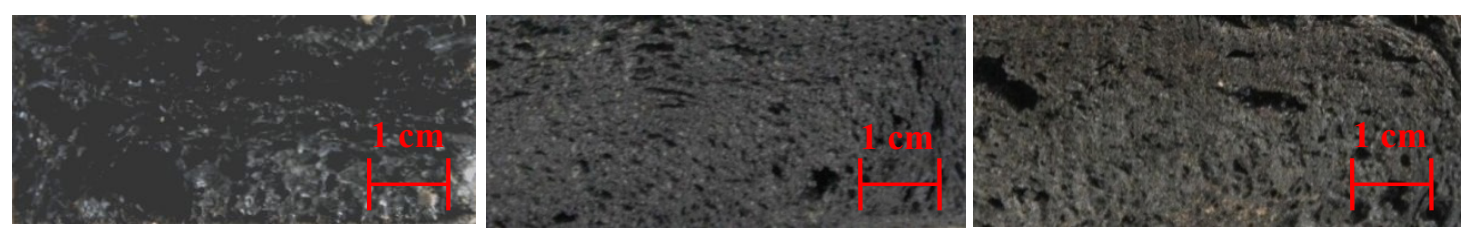

Figure 1. Appearance of the microwave-produced foams $\mathrm{A}\left(\mathrm{d}=110 \mathrm{Kg} / \mathrm{m}^{3}\right) ; \mathrm{B}\left(\mathrm{d}=150 \mathrm{Kg} / \mathrm{m}^{3}\right) ; \mathrm{C}\left(\mathrm{d}=224 \mathrm{Kg} / \mathrm{m}^{3}\right)$ (from left to right)
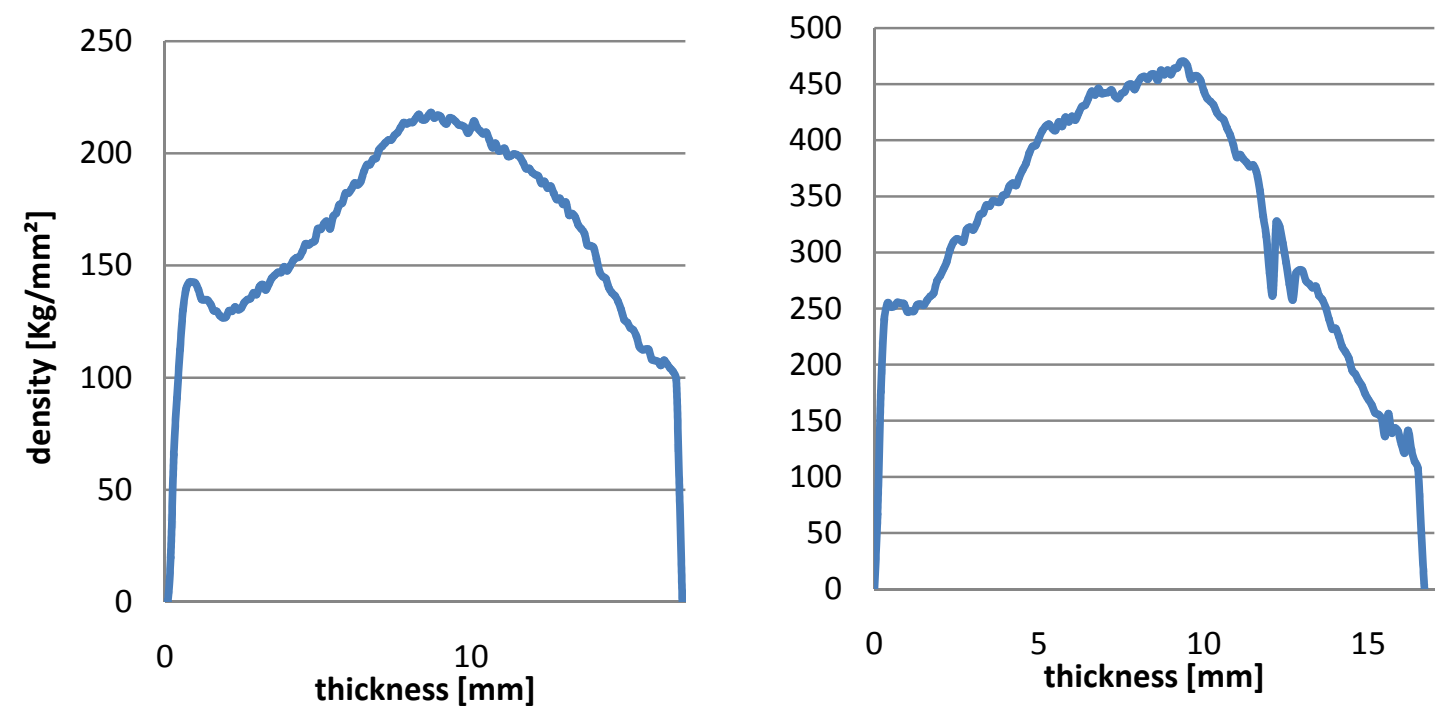

Figure 2. Density profile of the foam B (left) and C (right) with density of $150 \mathrm{Kg} / \mathrm{m}^{3}$ and $224 \mathrm{Kg} / \mathrm{m}^{3}$ respectively

The graphics show that the core of the foam is sufficiently homogeneous, while thereare low density zones in the borders. This effect is due to the pressure of the vaporsduring the blowing of the foam. Indeed, the blowing agents evaporate almostsimultaneously and the current of thesevapors creates preferential channels with bigger cells on the side of the structure.

Unfortunately these measurements cannot be applied for materials with densities lower than $150 \mathrm{Kg} / \mathrm{m}^{3}$ because this density is the minimal resolution of the instrument.

However the core of the foams can be considered homogeneous enough and mechanical, water and fire properties can be studied.

\subsection{Compression Resistance}

The compressive strength of tannin-based rigid foams is directly proportional to the density (Tondi \& Pizzi, 2009). Table 2 shows the behaviour of the microwave- produced tannin foams compared to the foams produced in the 
homologue pressand to the formaldehyde- reinforced ones.The general trend of the data reported in this table confirms that density plays a key role in the compression resistance.

Table 2. Average compressive strength of the three microwave-produced formulations and their standard deviations compared to the hot-press produced foams and to the formaldehyde reinforced foam

\begin{tabular}{cccc}
\hline Production & Density $\left(\mathrm{Kg} / \mathrm{m}^{3}\right)$ & $\begin{array}{c}\text { Compressive strength } \\
\left(\mathrm{N} / \mathrm{mm}^{2}\right)\end{array}$ & $\begin{array}{c}\text { Standard deviation } \\
\delta(\%)\end{array}$ \\
\hline Microwaves (A) & 110 & 0,04 & $50 \%$ \\
Microwaves (B) & 150 & 0,31 & $32 \%$ \\
Microwaves (C) & 224 & 0,33 & $45 \%$ \\
Hot Press & 90 & 0,12 & $15 \%$ \\
Hot Press & 143 & 0,16 & $19 \%$ \\
Hot Press & 240 & 0,44 & $9 \%$ \\
Reinforced & 100 & 0,35 & $(1)$ \\
Reinforced & 150 & 0,56 & $(1)$ \\
Reinforced & 200 & 0,76 & $(1)$ \\
\hline
\end{tabular}

Note 1: The data of the reinforced foams have been extrapolated by Tondi and Pizzi (2009)

However, the most significant information in this table is the high standard deviation of the measurements. This occurs because it is very difficult to control the energy distributionin a lab-scale microwave production and consequently tannin foams with similar bulk densities can have different mechanical propertiesbecause the cell distribution and dimension are different.

The synthesis with microwave produces unpredictable modifications in the cell arrangement caused by the immediate and severe evaporation of the blowing agents.

Not withstanding this technological knot, the microwave-produced tannin-furanic foams have similar mechanical properties to the hot-press produced foams. These foams are generally weaker than the formaldehyde-reinforced foams but they are still resistant enough to support the mechanical stress of $300 \mathrm{Kg}$ on a square surface of $10 \mathrm{~cm}$ by side.

An example of compression curve for $150 \mathrm{Kg} / \mathrm{m}^{3}$ microwaves-produced formaldehyde-free tannin foam is reported in Figure 3. The graphic shows two zones: The linear elastic region and the stress-plateau region.

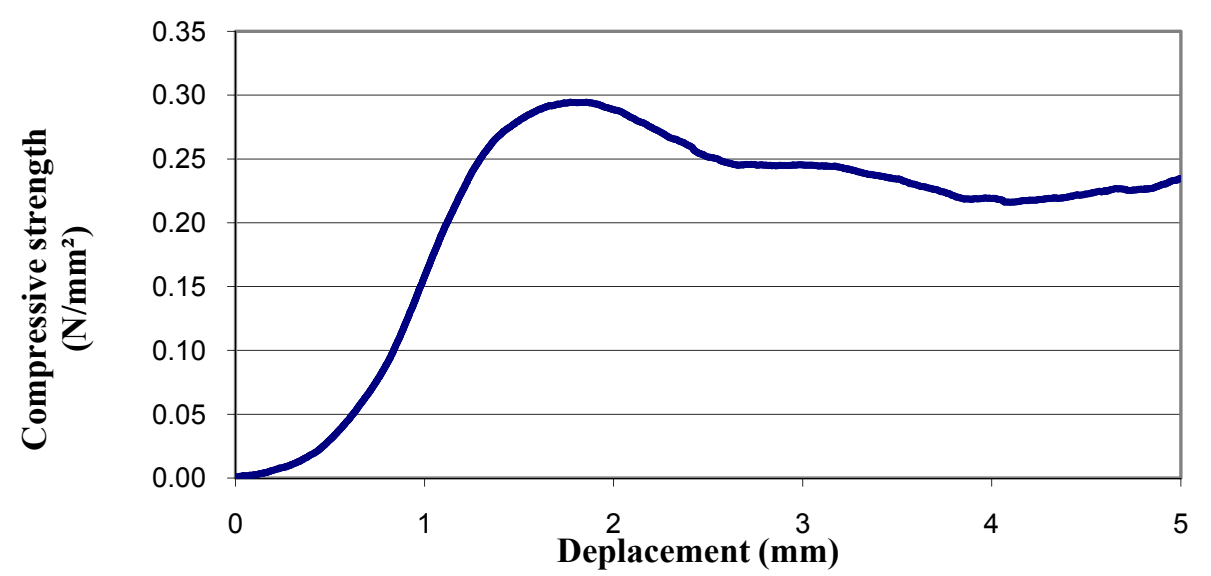

Figure 3. Example of compression curve for $150 \mathrm{Kg} / \mathrm{m}^{3}$ a microwaves-produced foam 
The slope of the linear elastic part of the curve is the Young's modulus; the stress reaches a peak value at the end of the elastic region and corresponds to the compressive strength or fracture stress. The maximum stress is often followed by a decrease before the stress-plateau is reached (post-yield softening) (Ashby, 2006). The stressed foam produces cracks when the fracture stress value is reached and the material tends to generate fragments as a result of these cracks.

\subsection{Moisture Absorption}

Another important feature for the rigid foams is their stability against humidity. Limited dimensional changes promotethe use of the material for construction. The tannin-furanic foams are very stable from the dimensional viewing angle: Length, height and width slightly increaseand the maximum volume increasefor $10 \%$ increase in relative humidity was $0.6 \%$.

In terms of weight increase the affinity towards water appears clearly in Figure 4 . Between 0 and $80 \%$ humidity, the moisture content constantly increases up to a value of $40 \%$. When the relative humidity rises over $80 \%$, the surface saturation point is exceeded; hence free water molecule sare rapidly stored in the cells. All foams show similar behaviour towards humidity, regardless of their density andproduction method.

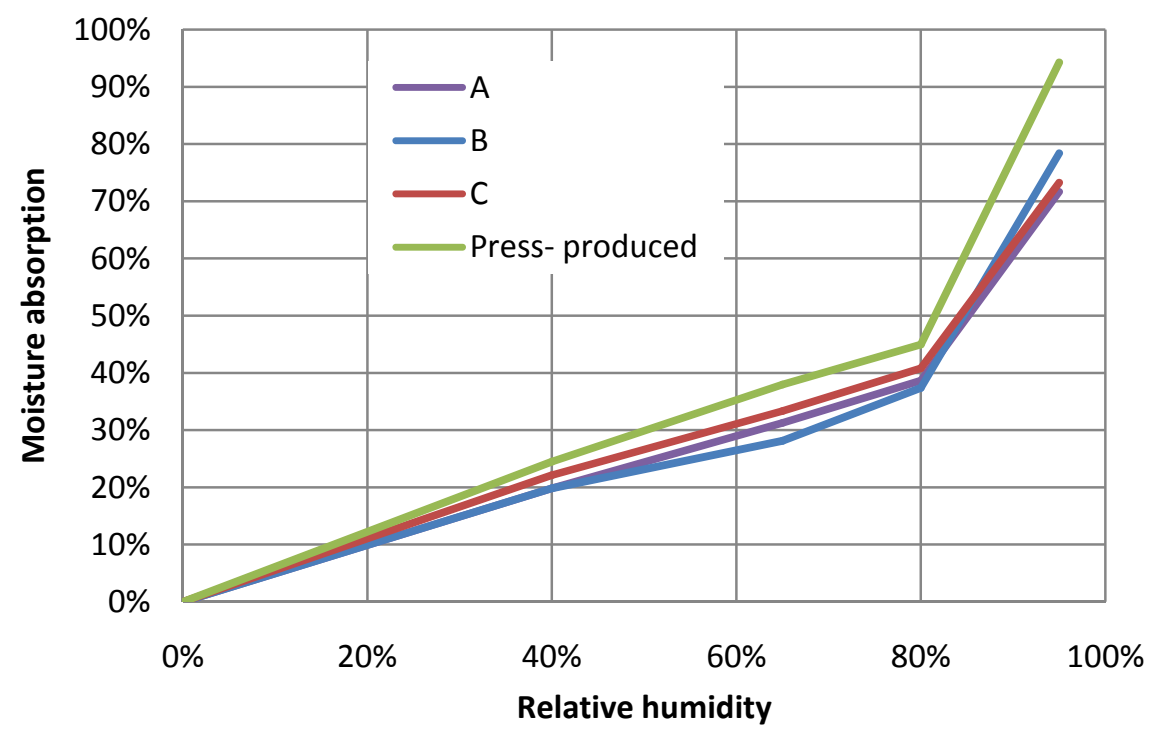

Figure 4. Moisture absorption of the microwave-produced foams compared with a hot-press produced foam $\left(240 \mathrm{Kg} / \mathrm{m}^{3}\right)$

\subsection{Water Absorption}

$0 \quad 20$ time [h] $30 \quad 40 \quad 50$ ter absorption reported in

Figure 5. It can be observed, that most of the foams absorb more than three timestheir weight.

Another interesting effect to be taken into consideration is the natural (non-forced) absorption.

It can be observed that the water absorption after 24 hours is very similar for each formulation and that the only difference is the time required to fill the foam completely.

The behaviour of similar foam with $180 \mathrm{Kg} / \mathrm{m}^{3}$ produced in the pressand forcedly immersed in water (Press produced forced of Figure 5) shows a comparable trend. This means that the tannin-furanic foams have similar behaviour in water regardless of their production method; therefore the cross-linking of the resin does not significantly affect the affinity between polymer and water. 


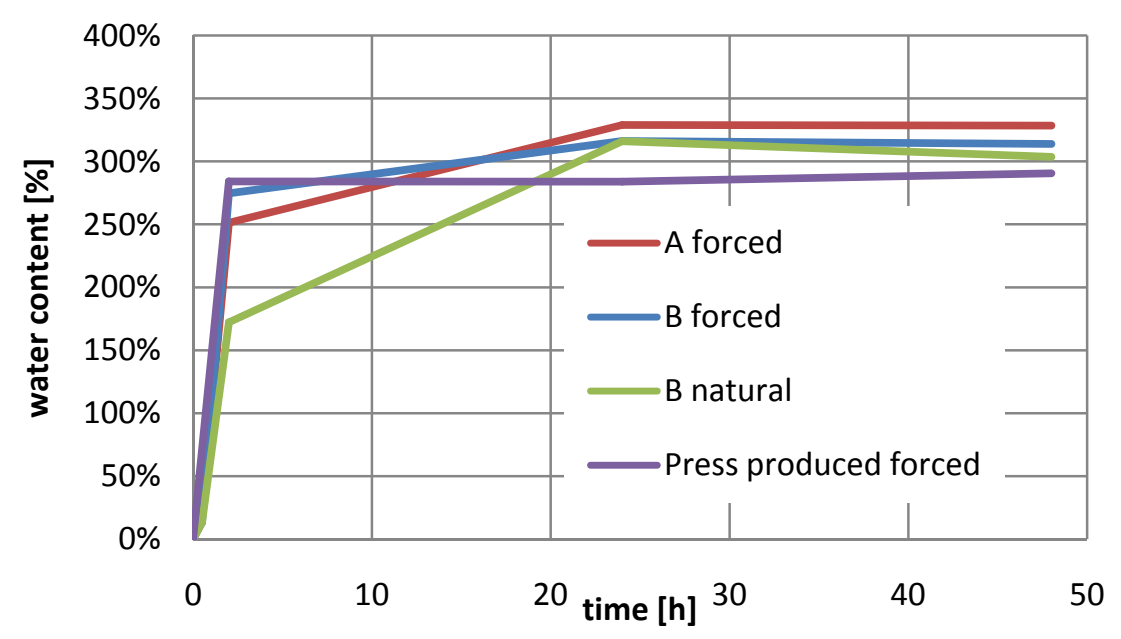

Figure 5. Water absorption of the microwave-produced foams compared with a hot- press produced foam (180 $\left.\mathrm{Kg} / \mathrm{m}^{3}\right)$

\subsection{Catalyst Recovery}

Tannin-furanic foams have a very high affinity towards water but when the polymer is cross-linkeditisabsolutely stable and it does not dissolvein water at all. For this reason it was possible to perform the catalyst recovery test. The tests have shown that the $\mathrm{pH}$ of the surface of the foam is rather acid (Table 3). The neutralization of the surface was carried out by washing the foams with water and the $\mathrm{pH}$ of this water allowed the calculation of the amount of catalyst that could be recovered.

It was possible for all the foams to recover between 7 and $8 \%$ of the original amount of catalyst used for the polymerization. This means that around $90 \%$ of the catalyst remains trapped in the network. This aspect of the process highlights its potential application in an industrial context.

Table 3. pH and catalyst recover after 4 leaching cycles

\begin{tabular}{ccccc}
\hline Foam & Density & Min $\mathrm{pH}$ & \% recover & Standard deviation \\
\hline A & 110 & 2,04 & 7,04 & 0,23 \\
B & 150 & 1,98 & 7,32 & 0,97 \\
C & 224 & 2 & 7,81 & 0,18 \\
\hline
\end{tabular}

\subsection{Fire Properties}

The most important characteristic of these tannin-furanic compounds is their high resistance to fire. The fire test performed facilitated the evaluation of specific fire-resistant properties: (i) the time the foams can glow after being exposed to 60 seconds of direct fire; (ii) the weight of the foam sample after glowing (Table 4).

It was noted that no-flamefeeding occurred for all the formulations. Furthermore, the foam A and C showed a very limited glowing time while foam B, produced with methanol as solvent, glowed for a longer period of time. All these results show an enhancement of the fire-proof properties of the foam that are at least comparable to the one obtained with the formaldehyde reinforced foams.

The auto-extinguish time is dependent on: (i) the surface area which is reached by oxygen during glowing, (ii) the thickness of the cell walls and (iii) the degree of polymerisation of the resin.

It could be determined that the flame in the samples with the high external surface area, thinner cell walls and lesspolymerized resins lasted longer.

However, the high fire resistance of the microwaves produced foamshas been also confirmed by the fact that around $80 \%$ of them was resisting after glowing. 
Table 4. Autoextinguishing time and remaining weight of the sample after direct flame exposure

\begin{tabular}{ccccc}
\hline Foam & $\begin{array}{c}\text { Density } \\
\left(\mathrm{Kg} / \mathrm{m}^{3}\right)\end{array}$ & $\begin{array}{c}\text { Autoextinguishing } \\
\text { time }(\mathrm{s})\end{array}$ & $\begin{array}{c}\text { Weight after } \\
\text { exposure }(\%)\end{array}$ & Appearance \\
\hline $\mathrm{A}$ & 110 & 48 & 82 & Core was intact \\
$\mathrm{B}$ & 150 & 283 & 78 & Core was intact \\
$\mathrm{C}$ & 224 & 93 & 80 & Core was intact \\
Hot Press & 240 & 50 & 82 & Core was intact \\
Form. Reinforced & 56 & 382 & - & Ashes \\
\hline
\end{tabular}

\section{Conclusion}

The method for tannin-furanic foam production affectsthe properties of the final product.The present study of the microwave production of tannin-furanic foams, demonstrated that:

- There is a possibility of producing homogeneous foams with tailored density in a limited time;

- Mechanical properties and water affinity between microwaves and press produced foams are comparable;

- Microwave-produced foams are fire-proof to a similar extent to the press-produced ones.

7 to $8 \%$ of the catalyst used for the polymerization can be recovered by water leaching and reused for the production of the material.

The microwave-produced foams are, indeed, very similar to those produced in the press. For this reason they can be considered more appropriate for industrial application because of their limited production time and for the limited amount of organic solvents required for blowing.

\section{Aknowledgements}

The authors gratefully aknowledge the Austrian Science Fund FWF. Projectsn. M. 1232-B16 and n.TRP 254-N13.

\section{References}

Aguilar, R., Ramırez, J. A., Garrote, G., \& Vazquez, M. (2002). Kinetic study of the acid hydrolysis of sugar cane bagasse. Journal of Food Engineering, 55, 309-318. http://dx.doi.org/10.1016/S0260-8774(02)00106-1

Ashby, M. F. (2006). The properties of foams and lattices. Philosophical Transacion Royal Society, 364, 15-30. http://dx.doi.org/10.1098/rsta.2005.1678

DIN 52185. (1976). German norm for the determination of the wood compression strength perpendicular to the grain.

EN317. (1993). European norm for the fiberboards. Determinationof swelling in thicknessafter immersion in water.

Link, M., Kolbitsch,C., Tondi, G., Ebner, M., Wieland, S., \& Petutschnigg, A. (2011). Formaldehyde-free tannin based foams and their use as lightweight panels. Bioresources, 6, 4218-4228.

Meikleham, N. E., \& Pizzi, A. (1994). Acid- and alkali-catalyzed tannin-based rigid foams. Journal of Applied Polymer Science, 53, 1547-1556. http://dx.doi.org/10.1002/app.1994.070531117

Nagaraja, B. M., Siva Kumar, V., Shasikala, V., Padmasri, A. H., Sreedhar, B., David Raju, B., \& Rama Rao, K. S. (2003). A highly efficient $\mathrm{Cu} / \mathrm{MgO}$ catalyst for vapour phase hydrogenation of furfural to furfuryl alcohol. Catalysis Communications, 4, 287-293. http://dx.doi.org/10.1016/S1566-7367(03)00060-8

Sealy-Fischer, V. J., \& Pizzi, A. (1992). Increased pine tannins extraction and wood adhesives development by phlobaphenes minimization. Eur. J. Wood. Prod., 50, 212-220.

Shi, S., \& Hwang, J. Y. (2003). Microwave-assisted wet chemical synthesis: advantages, significance, and steps to industrialization. Journal of Minerals and Material Characterization \& Engineering, 2(2), 101-110.

Tondi, G., Oo, C. W., Pizzi, A., Trosa, A., \& Thevenon, M. F. (2009a). Metal adsorption of tannin based rigid foams. Industrial Crops and Products, 29, 336-340. http://dx.doi.org/10.1016/j.indcrop.2008.06.006

Tondi, G., Pizzi, A., \& Olives, R. (2008). Natural tannin-based rigid foams as insulation material in wood construction. Maderas, 10, 219-227.

Tondi, G., \& Pizzi, A. (2009). Tannin based rigid foams: Characterization and modifications. Industrial crops and Products, 29, 356-363. http://dx.doi.org/10.1016/j.indcrop.2008.07.003

Tondi, G., Zhao, W., Pizzi, A., Du, G., Fierro, V., \& Celzard, A. (2009). Tannin - based rigid foams: a survey of chemical and physical properties. Bioresources Technology, 100, 5162-5169. 